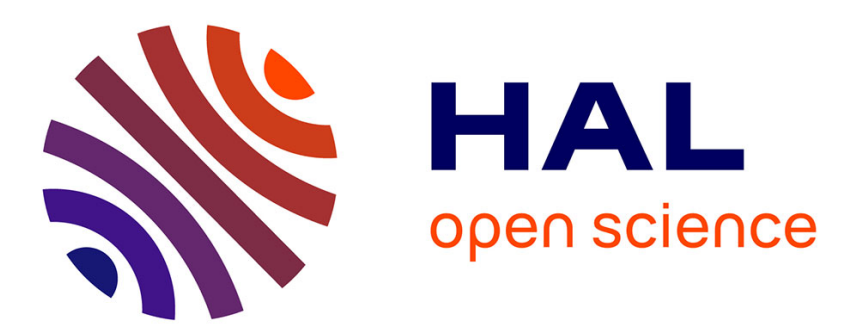

\title{
Utilization of stimulated Raman excitation and coherent anti-stokes Raman scattering in studies of bond- and mode-selective chemistry
}

\author{
T. Arusi-Parpar, Y. Cohen, D. David, I. Bar, S. Rosenwaks
}

\section{- To cite this version:}

T. Arusi-Parpar, Y. Cohen, D. David, I. Bar, S. Rosenwaks. Utilization of stimulated Raman excitation and coherent anti-stokes Raman scattering in studies of bond- and mode-selective chemistry. Journal de Physique IV Proceedings, 1994, 04 (C4), pp.C4-725-C4-728. 10.1051/jp4:19944201 jpa-00252663

HAL Id: jpa-00252663

https://hal.science/jpa-00252663

Submitted on 1 Jan 1994

HAL is a multi-disciplinary open access archive for the deposit and dissemination of scientific research documents, whether they are published or not. The documents may come from teaching and research institutions in France or abroad, or from public or private research centers.
L'archive ouverte pluridisciplinaire HAL, est destinée au dépôt et à la diffusion de documents scientifiques de niveau recherche, publiés ou non, émanant des établissements d'enseignement et de recherche français ou étrangers, des laboratoires publics ou privés. 


\title{
Utilization of stimulated Raman excitation and coherent anti-stokes Raman scattering in studies of bond- and mode-selective chemistry
}

\author{
T. ARUSI-PARPAR, Y. COHEN, D. DAVID, I. BAR and S. ROSENWAKS \\ Department of Physics, Ben-Gurion University of the Negev, Beer-Sheva 84105, Israel
}

\begin{abstract}
The state resolved photodissociation of rovibrational states of the fundamental symmetric stretch of water, $\mathrm{H}_{2} \mathrm{O}(1,0,0)$, and of the O-H or O-D stretch vibrations of $\mathrm{HOD}$ was studied using laser induced fluorescence and Doppler polarization spectroscopy. The control that the initially selected state exerts over the product state distribution, vector correlations and bond selectivity was demonstrated. The specific energy deposition via the lowest level of vibrational excitation leads to the enhancement of bond breaking and to structured rotational state distributions of the $\mathrm{OH}$ fragment which depends on the prepared rotational state of the parent. The correlations are close to the maximum attainable values expected for an idealized orientation where the transition dipole moment of the parent is parallel to the fragment angular momentum and perpendicular to its velocity. The photodissociation of HOD in which the $\mathrm{O}-\mathrm{H}$ stretch is initially excited, leads to enhanced bond breaking and selective production of OD + $\mathrm{H}$, while when the O-D is excited no enhancement is obtained. These results show that experiments which prepare the parent molecule in a particular state before a second laser dissociates it, provide a powerful means for bond- and mode- slective chemistry and molecular dynamics studies.
\end{abstract}

\section{1.- Introduction.}

Several experimental approaches have been introduced for studies of photodissociation of water from a selected initial state. Hausler $e t$ al. (1), in their pioneering experiment, have prepared a single rotational state of the antisymmetric stretch vibration by infrared excitation and photolyzed molecules in that state at $193 \mathrm{~nm}$ to determine the pathways of photodissociation from a single rovibrational state. Crim and coworkers $\left({ }^{2}\right)$ have extended this approach using vibrational overtone excitation thus allowing comparison among vibrational states, some having very similar energies but rather different nuclear motions. Using this method they demonstrated the control that the eigenstate from which the excitation occurs exerts on the dissociation. We have utilized stimulated Raman excitation (SRE) and coherent anti-Stokes Raman scattering (CARS) to prepare and detect, respectively, excited molecules in a particular rotational state of the symmetric stretch of water, $\mathrm{H}_{2} \mathrm{O}(1,0,0)$ and of the $\mathrm{O}-\mathrm{H}$ or $\mathrm{O}-\mathrm{D}$ stretch vibrations of HOD $\left(^{3}\right)$. Selective excitation of the parent molecule with one quantum of vibrational excitation is the cleanest possible way of investment of energy in a particular bond, since coupling to other vibrational modes is minimized for such excitation and often even specific rotational levels can be selectively pumped.

A complete understanding of the photodynamics of a molecule requires not only the consideration of overall scalar properties such as internal state distributions but also the vector properties such as product angular distribution and rotational alignment $\left({ }^{4}\right)$. Therefore, experimental photodissociation studies usually include measurements of scalar and vector observables. It is evident that measurements of vector properties in experiments where parent molecules are prepared in particular rotational states may provide additional insight into the dynamics of the dissociation events $\left(^{5}\right)$. This kind of measurement is essential since parent molecular rotation has been suggested as one of the reasons for decrease in the values of vector correlations.

The vector properties can be monitored via Doppler and polarization spectroscopy by exploiting the preferential absorbance of light by molecules with transition dipole moment, $\mu$, aligned parallel to the electric vector of the linearly polarized photolysis laser radiation, $\varepsilon_{\mathrm{p}}$. If the dissociation time is small compared to the rotational period of the parent, the alignment of $\mu$ may lead to recoil anisotropy (in the laboratory frame) of the photofragments, i.e., of the distribution of the recoil velocity, $v$, relative to $E_{p}$ (the so called $\mu-v$ correlation). In addition, fragment rotational alignment may be obtained as a result of 
an anisotropy in the distribution of the angular momentum, $\mathbf{J}$, relative to $\boldsymbol{E}_{\mathrm{p}}$ and thus to $\boldsymbol{\mu}$ (the $\boldsymbol{\mu}-\mathbf{J}$ correlation). Also, since both $J$ and $v$ are correlated with $\mu$, a mutual correlation between $v$ and $J$ (the $v$ $\mathbf{J}$ correlation and the triple $\boldsymbol{\mu}-\mathbf{v}-\mathbf{J}$ correlation) may result. The correlations, that can be extracted from the polarized Doppler broadened spectral line shapes and from time-of-flight spectra of the fragments, shed light on the motion of the photofragments in the molecular and laboratory frame and completely specify the photodissociation dynamics $\left({ }^{4}\right)$. To obtain all the vector correlations, we first measure the alignment parameter and then the polarized Doppler profiles. The Doppler profiles are measured at different laser geometries for different $\mathrm{OH}$ transitions, from which the various vector correlations are determined.

This paper describes the photodissociation dynamics of $\mathrm{H}_{2} \mathrm{O}$ and $\mathrm{HOD}$ excited to a particular rotational state, of the fundamental symmetric stretch and the O-H or O-D stretch vibrations, respectively. The motivation of this study is to control the photodissociation dynamics of molecules from particular rovibrational states, which are selected in the vibrational excitation step. The most important aspect is the preparation of distinct rovibrational states i.e. in-plane or out-of-plane rotations in $\mathrm{H}_{2} \mathrm{O}$. When an in-plane rotation is photodissociated, the measured vector correlations are close to the maximum values expected for an idealized orientation of $\mu$ parallel to the fragment angular momenta, $\mathbf{J}$, and perpendicular to the recoil velocity of the products, $v$.

\section{2.- Experiment.}

The experimental approach, shown in Fig. 1, involves excitation of a particular rovibrational state of $\mathrm{H}_{2} \mathrm{O}$ $(1,0,0)$, or HOD, photolysis of the vibrationally excited molecule at $193 \mathrm{~nm}$, and polarized laser induced fluorescence (LIF) detection of $\mathrm{OH}$. In the experiments which are conducted to determine the rotational distribution of the $\mathrm{OH}$, no polarization optics is used. The experimental approach and the apparatus have been described in detail elsewhere $\left(^{3}\right)$. Briefly, SRE and CARS are used to prepare and detect, respectively, particular rotational states of $\mathrm{H}_{2} \mathrm{O}(1,0,0)$. The excitation is effected with two visible beams, the second harmonic of a Nd:YAG laser at $532 \mathrm{~nm}$ and a tunable dye laser pumped by it, operating at $660 \mathrm{~nm}$. Both beams are parallelly polarized, collinearly combined and focused into the photodissociation region. The beams are precisely overlapped spatially and temporally. The frequency difference of the two lasers corresponds to excitation of the specific rotational state of $\mathrm{H}_{2} \mathrm{O}(1,0,0)$ through the isotropic Qbranch transition $\left({ }^{3}\right)$. The resulting CARS signal (which measures the SRE excitation and allows its optimization) is monitored by a photomultiplier tube (PMT) equipped with filters and imaging optics. Monitoring the CARS signal enables to assure constant level of excitation during the experiment.

Following the excitation, after $25 \mathrm{~ns}$, the rovibrational excited molecules are photodissociated by a $193 \mathrm{~nm}$ beam from an ArF excimer laser $(\sim 1 \mathrm{~mJ})$. The photodissociating beam is polarized by a quartz double prism Rochon polarizer (which allows the use of either $P$ (o-ray) or $S$ (e-ray) polarization direction, $E_{p}$ ) and then enters the reaction cell counterpropagating the SRE beams. The frequency doubled output beam of a Nd:YAG-pumped dye laser around $282.4 \mathrm{~nm}$ is used for LIF detection of the fragments after an additional delay of $25 \mathrm{~ns}$. The bandwidth of the probe laser is narrowed to $\sim 0.08 \mathrm{~cm}^{-1}$, in the UV, with an intracavity air-spaced angle-scanned etalon. The probe beam passes through an Archard-Taylor polarizer and a $\lambda / 2$ retarder which allows rotation of its polarization direction, $\varepsilon_{\mathrm{a}^{*}}$. The photolyzing and probe beam geometries could be altered to allow parallel or perpendicular alignment of the polarization $\left(\varepsilon_{\mathrm{p}} \| \varepsilon_{\mathrm{a}}, \varepsilon_{\mathrm{p}} \perp \boldsymbol{\varepsilon}_{\mathrm{a}}\right)$. The probe propagation direction $\left(\mathrm{k}_{\mathrm{a}}\right)$ is collinear with the SRE beams, thus counterpropagating the propagation direction of the photodissociating laser $\left(\mathbf{k}_{\mathrm{p}}\right)$. The LIF signal is detected at a right angle to the laser beams with a PMT equipped with an interference filter and imaging optics that is insensitive to the polarization. Water vapor is flowed at pressure of $100 \mathrm{mTorr}$ to minimize collisional relaxation of the initially prepared $\mathrm{H}_{2} \mathrm{O}$ state and of the rotationally excited $\mathrm{OH}$ fragments. In the HOD experiments, the sample is drawn from a liquid reservoir containing a statistical mixture of HOD, $\mathrm{H}_{2} \mathrm{O}$ and $\mathrm{D}_{2} \mathrm{O}$ with $\mathrm{H}: \mathrm{D}$ ratio of 1 .

\section{3.- Results.}

$\mathrm{H}_{2} \mathrm{O}(1,0,0)$.

The cross section for photodissociation from a particular rotation of $\mathrm{H}_{2} \mathrm{O}(1,0,0)$ at $193 \mathrm{~nm}$ is found to be $\sim 550$ times greater than that for $\mathrm{H}_{2} \mathrm{O}(0,0,0)$. Since this result has extensively been discussed in a 
previous publication $\left({ }^{3 a}\right)$ we will concentrate below in finer details of our studies. In the photodissociation process the $\mathrm{OH}$ radical is produced in the electronic ground state, where each rotational state is split into two spin-orbit states, $\Pi_{1 / 2}$ and $\Pi_{3 / 2}$, and to two $\Lambda$-doublet components, designated as $\mathrm{A}^{\prime}$ and $\mathrm{A}^{\prime \prime}$. Each rotational level is designated by $\mathrm{J}$ or $\mathrm{N}$, where $\mathrm{J}$ is the total angular momentum quantum number and $\mathrm{N}=\mathrm{J}+1 / 2$ and $\mathrm{J}-1 / 2$ for $\Pi_{3 / 2}$ and $\Pi_{1 / 2}$, respectively. In the high $\mathrm{J}$ limit (Hund case $b$ ) the $\mathrm{A}^{\prime}$ and $\mathrm{A}^{\prime \prime}$ states are symmetric and antisymmetric with respect to reflection in the plane of rotation of the

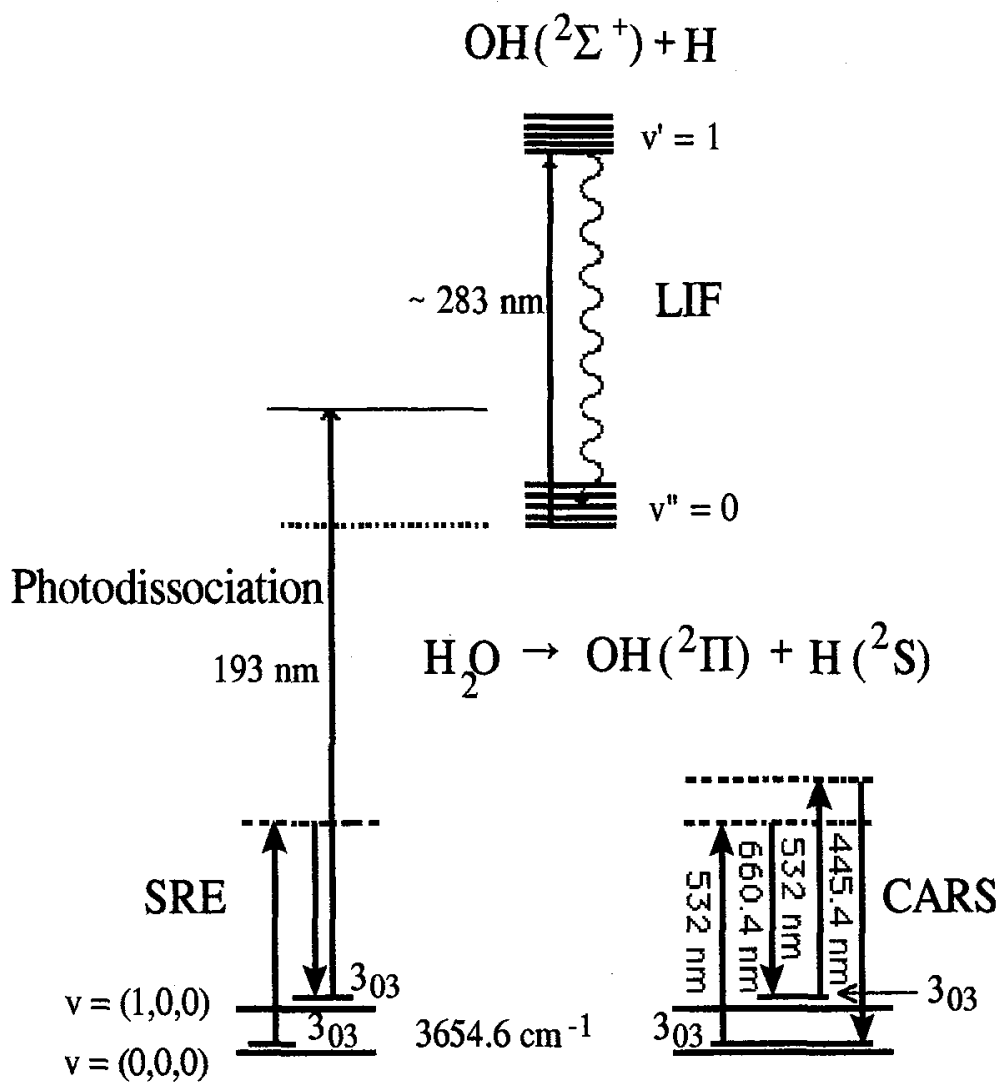

Fig. 1. Scheme of the experiment for the photodissociation of the $3_{03}$ rotational state of $\mathrm{H}_{2} \mathrm{O}(1,0,0)$. $3654.6 \mathrm{~cm}^{-1}$ corresponds to the excitation of the 303 rotational level of the ground vibrational state of $\mathrm{H}_{2} \mathrm{O}$ molecules to the $(1,0,0)$ state via SRE. The vibrationally excited state of the water molecule is subsequently photodissociated using $193 \mathrm{~nm}$ photons and the OH products are detected by LIF.

$\mathrm{OH}$. The $\mathrm{A}^{\prime}$ state is probed by the $\mathrm{P}$ and $\mathrm{R}$ branch transitions, while the $\mathrm{A}^{\prime \prime}$ by the $\mathrm{Q}$ branch transition In the photodissociation of $\mathrm{H}_{2} \mathrm{O}(1,0,0)$ only low $\mathrm{OH}$ rotational levels are populated and they have a structured distribution ${ }^{(3)}$ which agrees reasonably good with theory. Also, a N-dependent preferential population for the $A^{\prime \prime}$ component is observed, and the ratio $\Pi\left(A^{\prime \prime}\right) / \Pi\left(A^{\prime}\right)$ approaches values of $\sim 6$. The calculations predict higher values and much more pronounced structure of the $\Lambda$-doublet ratios than is observed in our experiments. The preference of the $A^{\prime \prime}$ component is attributed to the conservation of the electronic symmetry in the break-up of the excited transition-complex. The $\mathrm{A}^{1} \mathrm{~B}_{1}$ state of the dissociating molecule is antisymmetric, while the formed hydrogen atom is symmetric and therefore the $\mathrm{OH}$ must be formed in the antisymmetric A" state. However, assignment of the two $\Lambda$-doublets as the antisymmetric $A^{\prime \prime}$ and the symmetric $A^{\prime}$ states is a good approximation only for high N's $\left(^{3}\right)$. For low and intermediate $\mathrm{N}$ 's, A-doublet mixing occurs between the two components, leading to the $\mathrm{N}$-dependence preference. 
Strong N-dependent preference of the A" component has previously been found in the dissociation of cold $\mathrm{H}_{2} \mathrm{O}$ at 157 and $193 \mathrm{~nm}\left({ }^{2}\right)$.

$\mathrm{A}_{0}{ }^{(2)}$, the alignment parameter, is determined from the variation of the $\mathrm{OH}$ LIF intensities in two geometries where $\varepsilon_{\mathrm{a}} \perp \varepsilon_{\mathrm{p}}$ and $\varepsilon_{\mathrm{a}} \| \varepsilon_{\mathrm{p}}$. The values of $\mathrm{A}_{0}{ }^{(2)}$ as a function of $J$ are obtained from the ratio of the intensities of the $Q$ lines. The measured values of $A_{0}(2)$ increase with $J$ for dissociation from both the in-plane rotational state, $3_{03}$, and the mixed in- and out-of-plane state which contains the $3_{21}+3_{22}+4_{14}$ states, and show a similar trend to that obtained from theoretical predictions $(\%$. However, the degree of alignment obtained for the photodissociation of parent molecules prepared in the in-plane rotational state, is higher (a factor of two for $\mathrm{J}=5.5$ ) than in the mixed in- and out-of-plane state. Also, the experimental value of $\mathrm{A}_{0}{ }^{(2)}$ for the $3_{03}$ state at the highest $\mathrm{J}$ is in good agreement with the calculated value. These results may be explained classically by noting that the transition moment for the $193 \mathrm{~nm} \mathrm{~A} \leftarrow \mathrm{X}$ excitation of $\mathrm{H}_{2} \mathrm{O}$ is perpendicular to the $\mathrm{H}_{2} \mathrm{O}$ plane, hence molecules lying in a plane perpendicular to the direction of $\varepsilon_{\mathrm{p}}$ are preferentially dissociated ( $\left.{ }^{4}\right)$. After fragmentation, the $\mathrm{OH}$ products rotate in the $\mathrm{H}_{2} \mathrm{O}$ plane leading to the alignment of $J$ parallel to $\varepsilon_{\mathrm{p}}$ and to $\mathrm{A}_{0}(2)>0$. The $\mathrm{OH}$ transition moment, $\mu_{\mathrm{OH}}$, is perpendicular to the internuclear axis and perpendicular to or rotating in the $\mathrm{OH}$ plane of rotation for $\mathrm{Q}$ or P/R lines, respectively.

From the differences observed between the shapes of the Doppler profiles we extract the values of the bipolar moments, $-0.44 \pm 0.15,-0.42 \pm 0.22$ and $+0.34 \pm 0.20$, corresponding to the $\boldsymbol{\mu}-\mathbf{v}, \mathbf{v}-\mathrm{J}$ and $\boldsymbol{\mu}-\mathbf{v}-\mathbf{J}$ correlations, respectively. The limiting values of the bipolar moments expected for the idealized orientation of $\mu \| \mathbf{J} \perp \mathbf{v}$ at high $\mathrm{J}$ are $-0.5,-.0 .5$ and +0.5 , respectively. The observed moments approach the limiting values, suggesting that the dissociation is very close to being completely planar. As mentiomed above, parent in-plane rotation has no influence on the observed alignment while out-ofplane rotations result in a decrease of the alignment. The fact that the $\boldsymbol{\mu}-\mathbf{v}, \mathbf{v}-\mathbf{J}$ and $\boldsymbol{\mu}-\mathbf{v}-\mathbf{J}$ correlations, which have been measured for dissociation from the $3_{03}$ state, approach their limiting values support the statement that an in-plane rotation preserves the planarity of the dissociation.

Although the deviations of the measured correlations from the limiting values lie within the estimated uncertainties, all the measured values seem to be lower. Apparently, the slight deviation of the correlations that involve $J$ from the limiting values is mainly due to the low $J(J=4.5)$ at which they have been measured.

\section{HOD.}

The studies of the photodissociation of HOD prepared in particular rovibrational states in the fundamental stretching modes show that it is possible to control the branching ratio of the $\mathrm{OH}$ and $\mathrm{OD}$ fragments by specific selection of the fundamental vibrational wavefunctions. The results demonstrate that the bond cleavage does not necessarily occur on the weakened bond and agree with theoretical calculations. Detailed discussion of these studies can be found elsewhwere $\left({ }^{3 b}\right)$.

To conclude, the results presented in this paper show that it is possible to study fine details of molecular dynamics on a truly state-specific level and to avoid the averaging caused by the simultaneous dissociation of several quantum states.

\section{4.- References.}

I1/ D. Hausler, P. Andresen, and R. Schinke, J. Chem. Phys. 87, 3949 (1987).

121 R.L. Vander Wal, J.L. Scott, and F.F. Crim, J. Chem. Phys. 94, 1859 (1991); R.L. Vander Wal, J.L. Scott, and F.F. Crim, J. Chem. Phys. 92, 803 (1990).

13/ a. D. David, A. Strugano, I. Bar, and S. Rosenwaks, J. Chem. Phys. 98, 409 (1993); b. I. Bar, Y. Cohen, D. David, T. Arusi-Parpar, S. Rosenwaks and J.J. Valentini, J. Chem. Phys. 95, 3341 (1993).

14/ L.D.A. Siebbeles and J.A. Beswick, J. Chem. Soc. Faraday Trans. 88, 2565 (1992); and references therein.

15/ G.E. Hall and P.L. Houston, Ann. Rev. Phys. Chem. 40, 375. (1989).

16/ D. David, I. Bar, and S. Rosenwaks, J. Chem. Phys. 99, 4218 (1993). 